\title{
Cancer signaling: when phosphorylation meets methylation
}

\author{
Cell Research (2014) 24:1282-1283. doi:10.1038/cr.2014.103; published online 8 August 2014
}

The propagation of kinase-mediated phosphorylation signals is central to the oncogenic activity of the RAS-MAPK pathway in human cancers. A recent study shows that protein lysine methylation controls the phosphorylation status of a key component of the RAS-MAPK pathway to enable oncogenic KRAS in cancer progression.

RAS oncogene is the most frequently mutated oncogene in human cancers [1]. Over three decades ago, remarkable progress has illuminated the biochemical mechanisms and biological outcomes of oncogenic RAS. The biochemical and structural properties of this crucial oncogene have thwarted medicinal chemistry efforts to develop pharmacological inhibitors, thus directing concerted efforts towards targeting the RAS signaling components in the MAPK pathway. Such therapeutic strategies have shown relatively modest impact in RAS-driven tumors due in part to the complex feedback regulation of this signaling network and the general requirement of RAS signaling in normal tissue homeostasis [2]. Nearly all current small-molecule inhibitors targeting these RAS-MAPK pathway components function to inhibit their kinase activity which is critical for the phosphorylation and activation of their downstream effectors. While the major mode of regulation of these signaling components involves phosphorylation and dephosphorylation events, emerging data are uncovering other types of post-translational modifications (PTMs) with an equally important effect on protein activity and biological outcomes [3]. The targeting of these
PTMs, especially tumor-relevant PTMs, may therefore provide novel therapeutic avenues for cancer.

In exploring novel PTM of the RASMAPK signaling cascade, Mazur et al. [4] examined the potential role of lysine methylation signaling in oncogenic RAS-driven tumors by conducting an expression survey of 54 human lysine methyltransferases (KMTs) in pancreatic ductal adenocarcinoma (PDAC), a cancer type where KRAS is mutated in about $90 \%$ of cases. Five KMTs were found to be overexpressed in human PDAC, including SMYD3, MLL5, EZH2, SETD5 and WHSC1L1. Previous work demonstrated that EZH2 overexpression is linked to the disease aggressiveness and WHSC1L1 resides in an amplicon in human PDAC $[5,6]$. Muzur et al. focused on SMYD3 given that it acts in the cytosol where KRAS also functions and SMYD3 shows the most significant correlation with PDAC progression. To substantiate a role for SMYD3 in Kras-mediated tumorigenesis, Smyd3 was conditionally deleted in the classical $\mathrm{Kras}^{\mathrm{G} 12 \mathrm{D}}$-driven PDAC and lung cancer mouse models. In both models, Smyd3 deficiency suppresses the development of $\mathrm{Kras}^{\mathrm{G} 12 \mathrm{D}}$-induced tumor formation and significantly prolongs overall survival, supporting a role for Smyd3 in Ras-driven cancer development and progression. Importantly, reconstitution with wild-type Smyd3, but not a catalytic inactive mutant, restores the tumorigenic activity in Smyd3-deficient animals, providing crucial evidence that the enzymatic activity of Smyd3 is required for its oncogenic function.

To identify SMYD3 targets, 9000 potential substrates were screened on a protein array platform and, strikingly, the only robust methylation target of SMYD3 was MAP3K2. In addition, among the 11 KMTs tested, SMYD3 was the only KMT capable of modifying MAP3K2 and SMYD3 shows no detectable activity toward other components of the MAPK signaling pathway. Correspondingly, MAP3K2 methylation was reduced in Smyd3null tumor tissues and tumor cells with SMYD3 knockdown, indicating that SMYD3 is required to sustain MAP3K2 methylation in vivo. Loss-of-function studies demonstrated that SMYD3mediated MAP3K2 methylation is required for full activation of MAPK pathway through the direct modulation on MEK1/2 phosphorylation, a finding consistent with reduced ERK1/2 activation in SMYD3-deficient tumors relative to SMYD3 wild-type tumors driven by oncogenic Kras. Interestingly, SMYD3-mediated methylation of MAP3K2 does not alter its intrinsic kinase activity. Thus, to explore the mechanism of activation of MEK1/2 by methylated MAP3K2, Mazur et al. employed SLILAC-based quantitative proteomic screen to identify proteins that bound differentially to methylated and un-methylated MAP3K2 peptide. The screen and further biochemical validation revealed that MAP3K2 forms a complex with PP2A holoenzyme via its regulatory subunit PPP2R2A and this critical interaction is blocked by MAP3K2 methylation. Moreover, treatment of the PP2A inhibitor, cantharidin, had a similar effect to Smyd3 knockout in Kras-driven PDAC mouse model. Therefore, SMYD3-mediated methyla- 
tion of MAP3K2 inhibits the recruitment of PP2A and thus augments the phosphorylation/activation of MEK1/2 and the signaling output of MAPK pathway driven by Ras oncogene.

In summary, this very elegant study opens new vistas for tackling oncogenic KRAS-driven cancers though some outstanding issues remain to be addressed. SMYD3 was shown to be overexpressed in RAS-driven tumors. However, it is not clear whether its expression is regulated by Ras signaling. On the other hand, recent study indicated that the output of oncogenic Ras signaling needs to be amplified to induce tumor development in pancreatic cancer [7]. It is possible that the activation of MAPK through SMYD3-mediated MAP3K2 methylation serves to bring oncogenic Ras signaling above pathological threshold to ensure malignant progression. Second, the study focused on the role of SMYD3-mediated methylation on MAP3K2 in oncogenic Ras-driven tumors. However, the data indicated that such modification should also be functional in tumor cells with wild-type Ras. To expand the translational potential of SMYD3 targeting, further studies are needed to characterize the function of SMYD3-mediated methylation in tumors driven by other oncogenes such as EGFR and HER2 where Ras may function as an essential mediator in tumor development. Finally, while the development of second-generation small-molecule inhibitors of epigenetic proteins is rapidly emerging for cancer therapies, this study indicates the importance of auditing the activities of these compounds not only on their traditional chromatin targets but also on cellular proteins with integral roles in cancer. While the RAS oncogene itself is under heavy post-translational modification [8], it is now clear that other components of the RAS pathway are also regulated by PTMs other than phosphorylation. In addition to the study by Mazur et al., it has been previously shown that MEK2 and MEK6 are acetylated at key serine residues which block their phosphorylation and the subsequent activation of MAPK pathway $[9,10]$. However, how these PTMs are regulated by driver oncogenes and the biochemical and functional relationships between these modifications are less understood. Comprehensive studies are needed to catalog the global changes across these PTMs that are induced by oncogene activation or upon targeted therapy. Such characterization should illuminate the rational development of new combination strategies between traditional kinase inhibitors, such as MEK or PI3K inhibitors, and other PTM inhibitors in cancers driven by oncogenic RAS, the emperor of all oncogenes.

\section{Haoqiang Ying ${ }^{1}$, Ronald A DePinho ${ }^{2}$ \\ ${ }^{I}$ Department of Molecular and Cellular Oncol- ogy, ${ }^{2}$ Departments of Cancer Biology, University of Texas MD Anderson Cancer Center, Houston, TX 77030, USA \\ Correspondence: Ronald A DePinho \\ E-mail: rdepinho@mdanderson.org}

\section{References}

1 Pylayeva-Gupta Y, Grabocka E, Bar-Sagi D. Nat Rev Cancer 2011; 11:761-774.

2 Stephen AG, Esposito D, Bagni RK, et al. Cancer Cell 2014; 25:272-281.

3 Sims RJ 3rd, Reinberg D. Nat Rev Mol Cell Biol 2008; 9:815-820.

4 Mazur PK, Reynoird N, Khatri P, et al. Nature 2014; 510:283-287.

5 Li CH, To KF, Tong JH, et al. Gastroenterology 2013; 144:1086-1097.e9.

6 Tonon G, Wong KK, Maulik G, et al. Proc Natl Acad Sci USA 2005; 102:9625-9630.

7 Daniluk J, Liu Y, Deng D, et al. J Clin Invest 2012; 122:1519-1528.

8 Ahearn IM, Haigis K, Bar-Sagi D, et al. Nat Rev Mol Cell Biol 2012; 13:39-51.

9 Mukherjee S, Keitany G, Li Y, et al. Science 2006; 312:1211-1214.

10 Mittal R, Peak-Chew SY, McMahon HT. Proc Natl Acad Sci USA 2006; 103:1857418579. 